treatment-refractory anorexia nervosa: a phase 1 pilot trial. Lancet 381: 1361-1370.

Lozano AM, Lipsman N (2013). Probing and regulating dysfunctional circuits using deep brain stimulation. Neuron 77: 406-424.

Mayberg HS, Lozano AM, Voon V, McNeely HE, Seminowicz D, Hamani C et al (2005). Deep brain stimulation for treatment-resistant depression. Neuron 45: 651-660.

Neuropsychopharmacology Reviews (2014) 39, 250-252: doi: 10.1038/npp.2013.244

\section{Proteomic Biomarkers for Brain Disorders: Technical Considerations and Challenges}

Proteomic technologies are being used to identify fluid-based biomarkers for detection, progression, and therapeutic response in brain disorders. This commentary focuses on the technical obstacles and challenges involved in the discovery, evaluation, and validation of such markers.

Following the initial excitement in the mid-90s that proteomics would yield definitive blood patterns to characterize tumor types, the proteome was explored in brain disorders, using cerebrospinal fluid (CSF) and plasma, with the expectation that these biofluids would reflect disease state and evidence of drug action. Conflicting results from a decade of studies and an international Human Proteome Project (http://www.thehpp.org/) highlight the need to revisit basic principles: standardization of protocols and sample collection, reproducibility of technology platforms (Mattsson et al, 2013) and controlling for false positives.

The Neuroscience Steering Committee of the Biomarkers Consortium (http://www.biomarkersconsortium.org/) undertook a proteomic analysis of plasma and CSF samples collected in the Alzheimer's Disease Neuroimaging (ADNI) study. A multiplex Luminexbased immunoassay panel was used to analyze plasma and CSF samples from $A D$, mild cognitively impaired and control subjects at baseline and 1 year. Several markers differentiated patients from controls; three proteins were consistent with earlier CSF studies, supporting the potential of plasma markers as a screening tool (Soares et al, 2012). Unfortunately, variability in inter-assay performance can lead to nonreplicable findings. CSF findings from the same ADNI subjects using a subset of the same multiplex panel, replicated only a few previously reported protein differences (Siuciak et al, 2012). Comparisons of plasma vs CSF profiles using the same platform make clear that only in a few instances are analytes sufficiently correlated to allow the use of plasma as a proxy for CSF (Potter et al, 2012). Thus, even with the ADNI studies, where standardized sample and proteomic protocols were used, variation in specific multiplex immunoassay analyte findings limits the interpretation of the results.

An emerging strategy views broad proteomic profiling of samples as 'exploratory' to be followed by highly sensitive, specific and reproducible assays targeted to one or more specific analytes. At the current stage of development, no multiplex immunoassaybased approaches that target $>10$ analytes have proved sufficiently sensitive to detect beta-amyloid and tau in CSF at the level achieved when optimizing conditions to simultaneously measure these analytes (Kang et al, 2012). Mass spectrometric-based assays offer an unbiased discovery approach (Craft et al, 2013); studies are underway with the same CSF ADNI samples allowing for a unique contrast to the multiplex immunoassay approach. Informatics and pathway analysis approaches can also be used to analyze proteomic data, but there is a tension between application of such methodologies and reproducibility of measures within a proteomic platform. High costs ( $>\$ 500$ per sample) and limited aliquots (especially for CSF) are factors to consider in further attempts to replicate or rule out findings, which if true, could prove important.

To realize the potential of proteomics, it is critical to understand the limitations of the technology platforms (eg, its analytical performancesensitivity, specificity, precision, stability, and reproducibility) and set standards for replication and verification of assay findings to advance promising markers to clinical application. In December of 2013, the Biomarkers Consortium will sponsor a workshop (https://www.signup4.net/public/ap.aspx? $\mathrm{EID}=\mathrm{CSFP} 11 \mathrm{E} \& \mathrm{TID}=$ WhpjeshOarRyJD UAXwZjKg\%3d\%3d) focused on characterizing the CSF proteome and developing guidelines for use of technologies platforms to better identify reliable markers of brain disease.

Linda S Brady ${ }^{1}$ and William Z Potter ${ }^{2}$

${ }^{1}$ Division of Neuroscience and Basic Behavioral Science, National Institute of Mental Health, Bethesda, MD, USA; ${ }^{2}$ Senior Advisor to the Director, National Institute of Mental Health, Bethesda, MD, USA Email: Ibrady@mail.nih.gov

\section{FUNDING AND DISCLOSURE}

The authors declare that, except for income received from the NIMH, LSB has no financial interests to disclose. WZP currently receives compensation from $\mathrm{NIMH}$ as a senior consultant, serves on Advisory Boards for AgeneBio, Amgen, Ironwood, Lilly, MedAvante, Taisho, Takeda, and Theravance and has personal financial holdings from his previous employment at Merck.

Craft GE, Chen A, Nairn AC (2013). Recent advances in quantitative neuroproteomics. Methods 61: 186-218. Kang JH, Vanderstichele $H$, Trojanowski JQ, Shaw LM (2012; Simultaneous analysis of cerebrospinal fluid biomarkers using microsphere-based XMAP multiplex technology for early detection of Alzheimer's disease. Methods 56: 484-493.

Mattsson N, Andreasson U, Persson S, Carrillo MC, Collins S, Chalbot S et al (2013). CSF biomarker variability in the Alzheimer's Association quality control program. Alzheimers Dement $\mathbf{9}$ 251-261.

Potter WZ, Pickering EH, Immerman F, Kuhn M Siuciak J, Shaw L. Alzheimer's Disease Neuroimaging Initiative (ADNI), the Foundation for $\mathrm{NIH}$ Biomarkers Consortium CSF Proteomics Project Team (2012). Cerebrospinal fluid (CSF) vs plasmabased biomarkers in Alzheimer's disease (AD), mild cognitive impaired $(\mathrm{MCl})$ and age-matched healthy controls (HC) from the Alzheimer's Disease Neuroimaging Initiative (ADNI) cohort. Alzheimers Dement 8: P217.

Siuciak JA, Pickering EH, Immermann F, Kuhn M, Shaw L, Potter WZ. Alzheimer's Disease Neuroimaging Initiative (ADNI), the Foundation for $\mathrm{NIH}$ Biomarkers Consortium CSF Proteomics Project Team (2012). Cerebrospinal fluid (CSF) biomarkers in Alzheimer's disease (AD), mild cognitively impaired $(\mathrm{MCl})$ and age-matched healthy controls (HC) from the Alzheimer's Disease Neuroimaging Initiative (ADNI) cohort. Alzheimers Dement 8 P216-P217.

Soares HD, Potter WZ, Pickering E, Kuhn M, Immermann FW, Shera DM et al (2012). Plasma biomarkers associated with the apolipoprotein $E$ genotype and Alzheimer disease. Arch Neurol 69 $1310-1317$.

Neuropsychopharmacology Reviews (2014) 39, 252: doi:10.1038/npp.2013.202 